\title{
Resectable Digestive System Carcinoma
}

National Cancer Institute

\section{Source}

National Cancer Institute. Resectable Digestive System Carcinoma. NCI Thesaurus. Code C156909.

Digestive system carcinoma that is amenable to surgical resection. 